\title{
VIOLENCIA DE GÉNERO: EL PAPEL DE LA EMPATÍA Y EL PERDÓN SOBRE LA ACTITUD HACIA VOLVER CON LA EXPAREJA
}

\author{
Beatriz Montes-Berges \\ María Aranda \\ Universidad de Jaén \\ aranda@ujaen.es
}

https://doi.org/10.17060/ijodaep.2017.n1.v2.948

Fecha de Recepción: 8 Enero 2017

Fecha de Admisión: 1 Abril 2017

\section{RESUMEN.}

En la intervención con mujeres víctimas de violencia de género es fundamental minimizar el impacto de la experiencia sufrida y disminuir la posibilidad de retorno a la relación violenta. Para ello es clave trabajar sobre variables con efecto reparador sobre el proceso. Considerando el papel que se ha otorgado al perdón en otros contextos clínicos y la vinculación de la empatía con éste, el objetivo del presente estudio fue analizar la relación y capacidad predictiva de la empatía y el perdón en sus dos dimensiones (perdón a la situación y autoperdón, y perdón al otro) sobre la actitud de volver con la expareja. Participaron 17 mujeres de entre 26 y 60 años. Se encontró que la capacidad 0 incapacidad de separarse de las emociones de los demás (reverberación), influye en la actitud de volver o no con la expareja. Además, las participantes con mayor dificultad para perdonarse a sí mismas y a la situación, presentaban también una dificultad más elevada de separación emocional 0 reverberación.

Palabras clave: violencia de género, empatía, perdón, actitud hacia volver.

\section{ABSTRACT.}

Gender violence: Empathy and forgiveness role on the attitude toward returning with the expartner.

In the intervention with battered women, to minimize the impact of the experience and to diminish the possibility of a return to the violent relationship is quite important. To achieve this purpose, working on variables with a restorative effect on the process is the key. Considering the role of forgiveness in other clinical contexts, and the linking of empathy with it, the objective of the study was to analyze the relationship and predictive capacity of empathy and forgiveness (forgiveness of the situation and self-forgiveness, and forgiveness of the other) on the attitude of returning with the ex-partner. The study involved 17 women between 26 and 60 years. It was found that the ability or 
inability to separate from the emotions of others (reverberation), as empathic ability, influences the attitude of returning or not with the ex-partner. In addition, participants with greater difficulty in self forgiveness and forgive the situation had a higher difficulty of separation or reverberation.

Keywords: Gender violence, empathy, forgiveness, attitude toward return.

\section{ANTECEDENTES}

Existen diversas variables que pueden influir en el retorno a la relación en la que se ha producido violencia de género. Las teorías que tratan de explicarlo van desde aproximaciones más psicosociales -centradas principalmente en el proceso de toma de decisiones de la víctima-, a otras más psicológicas que analizan los efectos de las contingencias de reforzamiento dentro de un patrón cíclico de violencia sobre el estado emocional y psicológico de la víctima (Amor, Bohórquez y Echeburúa, 2006). Otros factores relacionados con la presión social, dificultades económicas (Anderson, 2003) o conductas de coacción y manipulación del maltratador funcionan como variables de riesgo (Amor et al., 2006).

Desde el punto de vista terapéutico, resulta fundamental minimizar el impacto de la experiencia sufrida y disminuir la posibilidad de retorno a la relación. Para ello es clave trabajar sobre variables con un importante impacto sobre el proceso de recuperación. Considerando el papel otorgado al perdón en otros contextos clínicos y la vinculación de la empatía con éste, el objetivo del estudio fue analizar el efecto que ambas variables tienen sobre la predisposición a retomar la relación con el maltratador en el caso de mujeres víctimas de violencia de género.

Una concepción tradicional del perdón lo define simplemente como "dejar pasar", con una idea implícita de "simplemente lo olvidaré" (Bloomfield y Fielder, 1983), esto implica una disminución de Ios sentimientos negativos -por ejemplo, resentimiento y enfado- hacia la persona transgresora y la aparición de sentimientos positivos -por ejemplo, compasión- hacia ella. Desde dicha perspectiva, perdonar implicaría una mayor probabilidad de retomar la relación. En la misma dirección, Hargrave y Sells (1997) definen la capacidad de perdonar en términos del esfuerzo para restaurar sentimientos de amor y confianza. Para ellos, el proceso supone dar la oportunidad de compensación, lograr que la víctima pueda percibir a su ofensor como no amenazante y poder analizar junto con el victimario la experiencia pasada y el daño provocado.

En contraposición a estas conceptualizaciones de perdón, McKay y Fanning (1991), explican que la intervención terapéutica basada en la compasión (perdón) se centra en tres aspectos: a) la comprensión de lo que ha pasado en términos meramente cognitivos, b) la aceptación de la situación y, c) la reafirmación de la persona en el presente, la anticipación de un futuro mejor, la superación del rencor y de las situaciones negativas. En este sentido, el perdón adquiría una función reparadora. En general, los resultados de distintos estudios sugieren que las intervenciones que promueven este tipo de perdón pueden llevar a reducir los efectos nocivos del "no-perdón" y producir grandes beneficios como incrementos en la autoestima y satisfacción con la vida (e.g., Maltby, Day y Barber, 2004). El perdón, además, correlaciona positivamente con indicadores de salud mental y negativamente con indicadores de estrés (Kaplan, Monroe-Blum y Blazer, 1994).

En el caso concreto de las mujeres que han sufrido violencia de género, el autoperdón tiene un efecto especialmente positivo en la reducción de los sentimientos de culpa. Es frecuente que éstos aparezcan asociados a las percepciones que tienen sobre sí mismas, a decisiones pasadas, a los sentimientos respecto al agresor, a la interiorización de los comentarios culpabilizadores provenientes del maltratador, los familiares o la sociedad (Echeburúa, Corral y Amor, 2001).

Algunas investigaciones han encontrado que existe mayor disposición a perdonar cuando se dan atribuciones benignas respecto de la conducta del otro (Hall y Fincham, 2006) y se tiene empa- 
tía hacia el ofensor (Rizkalla, Wertheim y Hodgson, 2008). Según estos autores, las personas que perdonan al agresor tienden a mostrar altos niveles de empatía. La empatía sería, pues, un predictor crucial del grado de perdón que la víctima siente hacia el ofensor. Otros autores argumentan que la empatía motiva a cambiar desde el foco propio de la experiencia dolorosa hacia el foco centrado en el otro, suponiendo el cuidado de las necesidades del ofensor (McCullough, Worthington y Rachal, 1997). En este sentido, la empatía parece más un factor de vulnerabilidad que de protección para la mujer víctima de violencia de género. Sin embargo, las conclusiones -aun escasas- sobre el papel de empatía y el perdón en violencia de género, probablemente dependan de la conceptualización empleada de la variable empatía.

Diversas corrientes presentan una conceptualización dual del constructo. Nummenmaa, Hirvonen, Parkkola y Hietanen (2008) distinguen entre empatía cognitiva y emocional, la primera se activa cuando somos capaces de asimilar y anticiparnos a lo que piensan o sienten los demás, la segunda se refiere al "contagio" de la emoción del otro. Davis (1980) propone un modelo de cinco componentes: a) cognitivos, referidos a la interpretación y comprensión de las emociones de otros; b) toma de perspectiva, que implica buscar una lógica comprensiva a la situación emocional de otros; c) fantasía o representación cognitiva de uno mismo en la situación de otros; d) preocupación empática, descrita como sentimientos de compasión, preocupación y cariño ante el malestar de otros; e) malestar personal o distrés que la persona experimenta al observar las vivencias negativas de los demás. Algunas décadas antes, Reik (1949) establecía una serie de fases en lo que denominaba proceso empático: a) identificación o pérdida de la consciencia del yo a través de la experimentación de lo que el otro siente; b) incorporación del otro en nuestro interior y asunción de sus experiencias como propias; reverberación, supone conocer el significado de lo que estamos sintiendo, para lo cual comienza a interactuar nuestro yo con el yo interiorizado del otro, d) separación, una vez realizado todo el proceso anterior, nos separamos de las emociones interiorizadas y usamos la razón, ganando distancia y permitiéndonos realizar un análisis objetivo.

Desde una perspectiva funcional, la empatía formaría parte no solo de la capacidad de entender las emociones de otros, sino también de manejar las propias. Como una parte facilitadora de las relaciones interpersonales, la empatía tiene valor en el constructo de la inteligencia emocional (Muñoz y Chaves, 2013). La empatía se constituye como una habilidad para percibir, evaluar y actuar de acuerdo con las emociones de los demás; también favorece la regulación emocional al tomar en cuenta el impacto de los propios actos sobre otros. También ayuda a la adaptación, pues permite desarrollar acciones sociales congruentes con el estado emocional, conductas e intenciones de los demás; en otras palabras, favorece actitudes de intuición, responsabilidad y conexión (Gilar, Miñano y Castejón, 2008; López Jiménez, Barrera Villalpando, Cortés Sotres, Guines y Jaime, 2011).

En resumen, un abordaje del perdón desde aproximaciones tradicionales podría poner a la mujer en una situación de vulnerabilidad y predisposición a reiniciar la relación con el maltratador, puesto que se entiende como una restauración de los sentimientos de amor y confianza. Sin embargo, existen otras aproximaciones que permiten hacer uso clínico y terapéutico del perdón aplicable en los casos en los que el fin último no es la reconciliación con la persona que comete la ofensa, sino la restauración del bienestar de la víctima. La empatía aparece íntimamente relacionada al perdón la mayoría de las investigaciones, le otorgan un papel facilitador en los procesos de perdón. La facilidad de comprensión de los pensamientos, emociones y actos de los demás, implicaría una predisposición a restaurar las relaciones dañadas. Desde otras perspectivas que ponen el foco en la empatía como la capacidad de comprensión de las propias, además de las ajenas, su función estaría más próxima a la inteligencia emocional. 


\section{OBJETIVOS}

El objetivo del estudio fue analizar la relación y capacidad predictiva de la empatía y el perdón hacia uno mismo y el otro- sobre la actitud de volver con la expareja. A partir de este objetivo se planteó como hipótesis que ambas variables explicarían parte de la varianza en las puntuaciones de la variable dependiente. Específicamente, esperábamos encontrar una relación inversa entre la disposición a perdonar y la actitud de volver con la expareja, entendiendo el perdón como un factor protector que implica reparación emocional, reconstrucción de la confianza en uno mismo, y separación cognitiva y emocional con el hecho o el agente causante del daño. Anticipar el funcionamiento de la empatía resulta bastante más complejo por la propia diversidad del constructo y la ausencia de estudios específicos en el ámbito de la violencia de género. Sí se esperaba encontrar una relación directa entre perdón y empatía, ésta última podría facilitar tanto el proceso de autoperdón, como el perdón a la situación y al otro. Respecto a la actitud para volver, un nivel muy elevado de empatía, comprensión e identificación emocional del otro, cuando el proceso de perdón no está bien definido, podría actuar incrementando la actitud de retorno a la relación.

\section{PARTICIPANTES}

En la investigación participaron 17 mujeres víctimas de violencia de género de edades comprendidas entre 26 y 60 años. Todas ellas estaban en la fase inicial de un programa de intervención grupal llevado a cabo por el Instituto Andaluz de la Mujer (Jaén). El resto de variables sociodemográficas quedan recogidas en la Tabla 1.

Tabla 1. Resumen de las variables sociodemográficas.

\begin{tabular}{|c|c|c|c|c|c|c|}
\hline Participante & Edad & $\begin{array}{c}\text { Estado } \\
\text { civil }\end{array}$ & $\begin{array}{c}\text { Nivel } \\
\text { estudios }\end{array}$ & Profesión & $\begin{array}{r}\text { Años } \\
V d G\end{array}$ & Tipo de violencia \\
\hline 1 & - & Soltera & Secundarios & $\begin{array}{c}\text { Auxiliar } \\
\text { administrativo }\end{array}$ & 7 & $\begin{array}{l}\text { Psicológica/Física } \\
\text { Sexual /Económica }\end{array}$ \\
\hline 2 & - & Separada & Primarios & Ama de casa & 15 & Psicológica \\
\hline 3 & - & Separada & Primarios & Ama de casa & 47 & $\begin{array}{l}\text { Psicológica/Física } \\
\text { Sexual /Económica }\end{array}$ \\
\hline 4 & 57 & Separada & Primarios & Ama de casa & 40 & Psicológica/Sexual \\
\hline 5 & 51 & Separada & Primarios & Ama de casa & 33 & $\begin{array}{l}\text { Psicológica/Física } \\
\text { Sexual /Económica }\end{array}$ \\
\hline 6 & 52 & Separada & Primarios & Ama de casa & 33 & $\begin{array}{l}\text { Psicológica/Física } \\
\text { Sexual /Económica }\end{array}$ \\
\hline 7 & 50 & Casada & Secundarios & $\begin{array}{c}\text { Auxiliar } \\
\text { administrativo }\end{array}$ & 33 & $\begin{array}{l}\text { Psicológica/Física } \\
\text { Sexual /Económica }\end{array}$ \\
\hline 8 & 60 & Separada & Primarios & Ama de casa & 30 & Psicológica/Física \\
\hline 9 & - & Separada & Primarios & Jubilada & 36 & $\begin{array}{l}\text { Psicológica/Física } \\
\text { Sexual /Económica }\end{array}$ \\
\hline 10 & 46 & Divorciada & Primarios & Funcionaria & 14 & $\begin{array}{l}\text { Psicológica/Física } \\
\text { Sexual /Económica }\end{array}$ \\
\hline 11 & 35 & Casada & Secundarios & $\begin{array}{c}\text { Auxiliar } \\
\text { administrativo }\end{array}$ & 6 & Psicológica/Económica \\
\hline
\end{tabular}




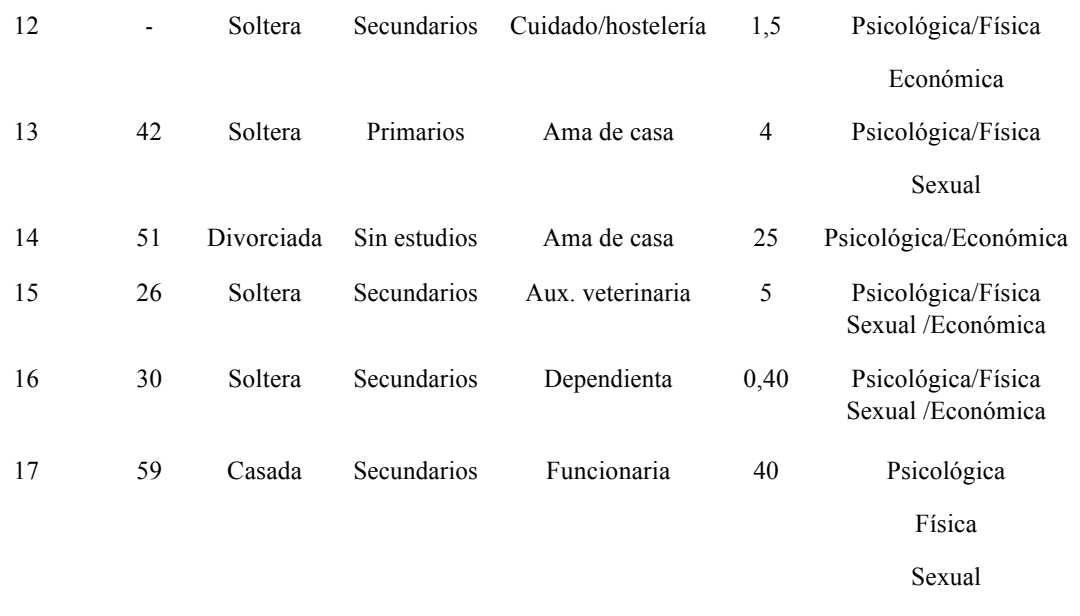

\section{METODOLOGÍA E INSTRUMENTOS}

Escala de Personalidad Émpata (EPE, Olmedo y Montes-Berges, 2011). Consta de cuatro subescalas y un total de 30 ítems. Se emplearon en el presente estudio las tres que se describen a continuación. Subescala de sentimientos $(2,4,5,7,9,13,14,16,19)$ evalúa la habilidad para detectar los sentimientos de los demás o identificación (ej. "Cuando estoy con otra persona capto sus sentimientos"), la introyección o capacidad de experimentar con la misma intensidad y claridad las emociones que provienen de otros (ej. "Siento una alegría desmesurada cuando tengo cerca a una persona que se siente muy feliz"), y proyección de emociones a los demás (ej. "A menudo hay personas que estando conmigo se sienten reconfortadas"). La fiabilidad de este factor fue de $\alpha=.50$. Subescala de características ápatas $(15,20,22,25-28,30)$, describe algunas características de lo ápatas como la imposibilidad o dificultad de sentir o experimentar las emociones de los demás (ej. "No me importa perjudicar a alguien si yo salgo beneficiado/a"). Este factor La fiabilidad obtenida fue de $\alpha=.82$. Subescala de separación o reverberación $(8,11,23)$, hace referencia a la influencia de las emociones de los demás y la imposibilidad o dificultad que tienen las personas émpatas de separarse de ellas (ej. "Me resulta difícil separarme de los sentimientos de los demás"). La fiabilidad de este factor fue de $\alpha=.67$. A más puntuación, menos capacidad de separación o reverberación.

Escala de actitud para perdonar (Montes-Berges, Vázquez, Aranda, Mora y Armenteros, manuscrito en elaboración). Está compuesta por 11 ítems, con respuestas tipos Likert de 5 puntos, desde 1 ("totalmente en desacuerdo") hasta 5 ("totalmente de acuerdo"). A mayor puntuación, menor capacidad de perdonar. El instrumento presenta dos dimensiones, perdón hacia uno mismo y la situación, e.g., "No paro de criticarme por los fallos que he cometido en mi vida" (1, 2, 4-7, 9-11), y perdón hacia el otro, e.g., "Cuando una persona me hace daño, sigo pensando mal de ella durante bastante tiempo" $(3,8)$. El alfa de Cronbach de las subescalas fue de $\alpha=.857, \alpha=.450$, respectivamente.

Actitud hacia volver con la expareja (Aranda, Vázquez, Montes-Berges, Mora y Armenteros, manuscrito en elaboración). El instrumento consta de 12 ítems con respuestas tipos Likert de 5 puntos, desde 1 ("totalmente en desacuerdo") hasta 5 ("totalmente de acuerdo"). A mayor puntuación, mayor actitud hace volver con la expareja. La fiabilidad alcanzó un valor de $\alpha=.927$. 
Cuestionario de variables sociodemográficas. Se recogió información sobre la edad, estado civil, profesión, nivel de estudios, tipo de violencia sufrida, número de años que ha sufrido maltrato y tipo de violencia (física, psicológica, sexual y económica).

\section{RESULTADOS}

Los principales descriptivos de las tres variables analizadas, empatía, perdón y actitud para volver con la expareja quedan recogidos en la Tabla 2. Respecto a la actitud para perdonar, se encontró una mayor dificultad para ejercer el autoperdón y el perdón a situaciones pasadas, $M=3.87$, en comparación con la capacidad de perdonar a la persona que cometió la infracción o daño, $M=2.79$. De las tres dimensiones de la empatía evaluadas, los valores más altos se alcanzaron en la capacidad de separación o reverberación, $M=4$.19. Finalmente, la media obtenida en la variable "actitud para volver con la expareja", mostró una predisposición baja de las participantes a retomar la relación.

Tabla 2. Descriptivos de las variables "perdón", "empatía" y "actitud para volver con la expareja"

\begin{tabular}{lllll}
\hline & Mínimo & Máximo & Medía & Dt \\
\hline Actitud volver & 1.00 & 2.73 & 1.43 & 0.69 \\
Perdón yo, situación & 2.50 & 4.80 & 3.87 & 0.72 \\
Perdón otro & 1.00 & 4.00 & 2.79 & 1.00 \\
Empatía sentimientos & 1.00 & 4.00 & 2.43 & 1.00 \\
Empatía reverberación & 2.33 & 5.00 & 4.19 & 0.83 \\
Empatía ápata & 1.57 & 2.71 & 2.24 & 0.32 \\
\hline
\end{tabular}

En el análisis de regresión empleado para evaluar la relación de las variables con la actitud de volver con la expareja se empleó en método de pasos sucesivos. En el modelo final, constituido en un solo paso, fueron eliminadas las dos dimensiones de perdón (perdón hacia una misma y la situación, perdón al otro), así como las subesalas de sentimientos y ápata de la EPE, todas las " $p$ " fueron mayores de .243. La subescala de reverberación fue la variable incorporada al modelo, con un coeficiente de regresión parcial significativamente distinto de cero al \%5. La capacidad de reverberación o separación explica un $27 \%\left(R^{2}=.27\right)$ de la variabilidad observada en la variable "actitud para volver con la expareja", $F(1,16)=5.56, p=.032$. Los coeficientes de regresión parcial que constituyen el modelo indican que la variable contribuye significativamente a mejorar la calidad del modelo de regresión $t=2.36, p=0.32, \beta_{1}=.393$, el coeficiente estandarizado de fue de .520 .

El estudio del efecto de la empatía sobre el perdón se llevó a cabo mediante ANOVAs. Previamente, se calcularon los niveles altos y bajos de las puntuaciones de las tres subescalas de empatía a partir de los percentiles 25 y 75 . En las tres dimensiones, la prueba T para muestras independientes, asumiendo varianzas distintas, mostró que las medias de los grupos, altos y bajos, fueron significativamente diferentes: a) subescala de sentimientos, $t(4.13)=-10.47, p=.000$; b) subescala de reverberación, $t(3.00)=-5.19, p=.014$; subescala ápata, $t(5.92)=-8.88, p=.000$. Finalmente, el ANOVA mostró que las participantes con más dificultades en capacidad de reverberación o separación obtenían valores significativamente más elevados en perdón hacia la situación 
y autoperdón, es decir, mayores dificultes en esta dimensión del perdón, $F(2,16)=6.45, p=.010$, $M=3, M=4.18$ (Figura 1).

Figura 1. Gráfica de medias del perdón a la situación y autoperdón en función del nivel alto y bajo en reverberación.

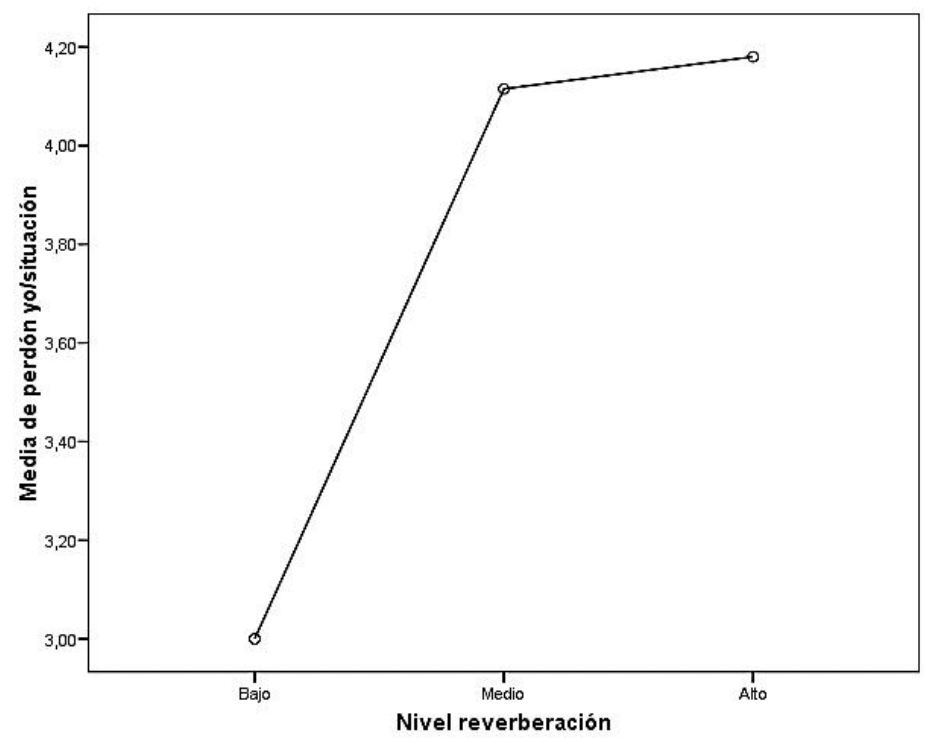

\section{DISCUSIÓN}

El objetivo del presente estudio fue analizar el papel que el perdón y la empatía juegan sobre la predisposición a retomar la relación en el caso de mujeres que han sido víctimas de violencia de género. Los análisis descriptivos de las variables mostraron una mayor dificultad de las participantes en el perdón hacia sí mismas y a las situaciones negativas vividas, en comparación con la capacidad de perdonar a la persona que comete el daño. Las puntuaciones relacionadas con la habilidad para detectar, identificar y experimentar los sentimientos de los demás (subescala de sentimientos) y la imposibilidad o dificultad de sentir o experimentar las emociones de los demás (ápatas), fueron bajas y similares entre ellas. En comparación con estudios de previos que emplearon la misma medida de autoinforme -la EPE-, ambas puntuaciones quedan por debajo de la media, que suele estar en torno a 3.3 para los hombres y 3.5 para las mujeres (Aranda, Montilla-Jiménez y MontesBerges, 2011; Olmedo y Montes-Berges, 2011). La escasez de datos sobre empatía en las mujeres que experimentan violencia de género, hace difícil encontrar un sustento empírico que permita explicar los resultados. A nivel general, estos datos difieren de las conclusiones de investigaciones que encuentran elevados niveles de empatía en las mujeres (e.g., Baron-Cohen, 2003), si bien es cierto que la población de referencia dista mucho de las concreciones asociadas a las mujeres víctimas de maltrato.

Las puntuaciones más elevadas se obtuvieron en la subescala denominada "reverberación". Esto implica que las participantes mostraban dificultad para separarse de la influencia de las emociones de los demás. Los valores se encuentran, de hecho, por encima de los datos obtenidos en otras investigaciones (Aranda et al., 2011; Olmedo y Montes-Berges, 2011). Esta variable fue la 
única significativamente relacionada con la actitud de volver con la expareja. No se encontró relación, sin embargo, entre perdón y la variable dependiente.

Si bien es cierto que un gran número de investigaciones otorgan al perdón una función importante en la reparación de las relaciones, en los casos en los que el foco no se encuentra en el ofensor -o en la relación- sino en la persona dañada, el perdón dejaría de estar relacionado con esa variable. En esta línea, Andrews (2000) considera el perdón como un proceso que se completa enteramente en el individuo dañado; no necesita de nada ni depende de la posición del agresor. El acto de perdón es totalmente independiente de las acciones del agresor, en el pasado, en el presente y en el futuro; en este sentido, se puede entender como un acto "completo". Cuando una persona perdona de esta manera, no busca nada del otro, ni en la práctica ni en teoría. Otros autores sostienen que, si bien el perdón hace más probable la reconciliación, no desemboca necesariamente en ella: se puede permanecer en una relación sin que haya habido perdón o bien perdonar a alguien con quien ya no se tiene ningún vínculo (e.g., Fincham et al., 2006; Gordon y Baucom, 2003). Por su parte, la formulación inicial de McCullough et al., (1998), ampliamente utilizada en las investigaciones empíricas, propone una concepción unidimensional del perdón: el descenso en la motivación a evitar y a vengarse del ofensor serían suficientes para considerar que alguien ha perdonado a otro.

Los resultados arrojaron también que las participantes con más dificultades en capacidad de reverberación o separación emocional mostraban mayores dificultes en autoperdón y perdón a la situación. Investigaciones previas han puesto de relieve el papel de la empatía como predictor del grado de perdón que la víctima siente hacia el ofensor (Prieto-Ursúa et al., 2012). Pero, ¿funcionaría esa misma relación respecto al perdón hacia una misma y la situación vivida? Probablemente, el foco habría que dirigirlo hacia la función de la empatía como parte de la inteligencia emocional, el reconocimiento y regulación de las propias emociones es un paso impredecible para la resolución de conflictos, las relaciones interpersonales, la toma de decisiones, etc. (Gilar et al., 2008; López Jiménez et al., 2011); por ende, cuando hay dificultad en esta capacidad, también se verán mermados los procesos posteriores.

\section{CONCLUSIONES}

El perdón es una variable sumamente compleja y con un gran potencial terapéutico, sin embargo, dependiendo de la naturaleza de la situación, relación o daño se requiere un tipo de perdón distinto. En el caso de mujeres víctimas de violencia de género, el perdón "unilateral” -entendiéndose como liberación del malestar producto del no perdón sin tener como objetivo la restauración de la relación- sería el más oportuno. De igual forma, el abordaje que se hace sobre el alcance de la empatía muestra también una gran heterogeneidad en los resultados, siendo relevante establecer la perspectiva desde la que se aborda su papel terapéutico. Lo que queda claro es que el nivel de empatía (más o menos elevado) no parece tan relevante como el manejo que la persona haga de la misma. En la medida en la que la víctima sea capaz de identificar y separar sus emociones de las de la otra persona, aumenta también la capacidad de autoperdón y los procesos de toma de decisiones, como el retorno o no a la relación.

\section{REFERENCIAS}

Amor, P., Bohórquez, I.A. y Echeburúa, E. (2006). ¿Por qué y a qué coste físico y psicológico permanece la mujer junto a su pareja maltratadora? Acción Psicológica, 4(2), 129-154.

Anderson, D.J. (2003). The impact on subsequent violence of returning to an abusive partner. Journal of Comparative Family Studies, 34, 93-112.

Andrews, M. (2000). Forgiveness in context. Journal of Moral Education, 29(1), 75-86. 
Aranda, M., Montilla-Jiménez, G. y Montes-Berges, B. (2011). Escala de personalidad émpata (EPE). En B. Montes-Berges (Ed.), Émpatas: la capacidad de sentir como la otra persona (pp. 93-132). Granada: Ruiz de Aloza, S.L.

Baron-Cohen, S. (2003). La gran diferencia. Cómo son realmente los cerebros de hombres y mujeres. Barcelona: Editorial Amat.

Bloomfield, H.H. y Fielder, L. (1983). Making Peace with Your Parents. New York: Ballantine.

Davis, M.H. (1980). A Multidimensional Approach to Individual Differences in Empathy. JSAS Catalog of Selected Documents in Psychology, 10, 85.

Echeburúa, E., Corral, P. y Amor, P.J. (2001). Estrategias de afrontamiento ante los sentimientos de culpa. Análisis y Modificación de Conducta, 27, 905-929.

Fincham, F.D., Hall, J. y Beach, S.R. (2006). Forgiveness in marriage: current status and future directions. Family Relations, 55, 415-422.

Freedman, S.R y Enright, R.D. (1996). Forgiveness as an intervention goal with incest survivors. Journal of Consulting and Clinical Psychology, 64(5), 983-992.

Gilar, R., Miñano, P. y Castejón, J.L. (2008). Inteligencia Emocional y empatía: su influencia en la competencia social en educación secundaria obligatoria. SUMA Psicológica UST, 5(1), 21-32.

Gordon, K.C. y Baucom, D.H. (2003). Forgiveness and marriage: preliminary support for a measure based on a model of recovery from a marital betrayal. The American Journal of Family Therapy, 31, 179-199.

Hargrave, T.D. y Sells, J.N. (1997). The development of a forgiveness scale. Journal of Marital and Family Therapy, 23, 41-62.

Hall, J.H. y Fincham, F.D. (2006). Relationship dissolution following infidelity: The roles of attributios and forgiveness. Journal of Social and Clinical Psychology, 25, 508-522.

Kaplan, B.H., Monroe-Blum, H. y Blazer, D.G. (1994). Religion, health and forgiveness. En J.S. Levin (Ed.), Religion in Aging and Health (pp. 52-77). Thousand Oaks, CA: Sage.

López Jiménez, M.T., Barrera Villalpando, M.I., Cortés Sotres, J.F., Guines, M. y Jaime, M. (2011). Funcionamiento familiar, creencias e inteligencia emocional en pacientes con trastorno obsesivo-compulsivo y sus familiares. Salud Mental, 34, 111-120.

Maltby, J., Day, L. y Barber, L. (2004). Forgiveness and mental health variables:Interpreting the relationship using an adaptational-continuum model of personality and coping. Personality and Individual Differences, 37, 1629-1641.

McCullough, M.E., Rachal, K.C., Sandage, S.J., Worthington, E.L., Brown, S.W. y Hight, T.L. (1998). Interpersonal forgiving in close relationships. II: Theoretical elaboration and measurement. Journal of Personality and Social Psychology, 75, 1586-1603.

McCullough, M.E., Worthington Jr., E.L. y Rachal, K.C. (1997). Interpersonal forgiving in close relationships. Journal of Personality and Social Psychology, 73, 321-336.

McKay, M. y Fanning, P. (1991). Autoestima. Evaluación y mejora. Barcelona: Martínez Roca.

Muñoz, A.P. y Chaves, L. (2013). La empatía: ¿un concepto unívoco? Katharsis, 16, 123-143.

Nummenmaa, L., Hirvonen, J. Parkkola, R. y Hietanen, J.K. (2008). Is emotional contagion special? An fMRI study on neural systems for affective and cognitive empathy. Neuroimagen, 43(3), 571580.

Olmedo, P. y Montes-Berges, B. (2001). Análisis de la empatía en la violencia de género. Trabajo Tutelado de Investigación no publicado.

Prieto-Ursúa, M., Carrasco Galán, M.J., Cagigal de Gregorio, V., Gismero González, E., Martínez Díaz, M.P. y Muñoz San Roque, I. (2012). El Perdón como Herramienta Clínica en Terapia Individual y de Pareja. Clínica Contemporánea, 3(2), 121.134. doi: 10.5093/cc2012a8 


\section{EXPAREJA}

Reik, T. (1949). Listening with the third ear. Nueva York: Farrar, Strauss \& Co. izkalla, L., e r t heim, E.. y odgson, L.. (2008).he roles of emotion management and perspective taking in individuals conflict management styles and disposition to forgive. Journal of Research in Personality, 42, 1594-1601.

Worthington Jr., E. L. y Wade, N. G. (1999). The psychology of unforgiveness and forgiveness and implications for clinical practice. Journal of Social \& Clinical Psychology, 18, 385-418. 